\title{
Covert Communication in MIM0-OFDM System Using Pseudo Random Location of Fake Subcarriers
}

\author{
Rizky Pratama Hudhajanto, I Gede Puja Astawa, Amang Sudarsono \\ Graduate School of Electrical Engineering \\ Politeknik Elektronika Negeri Surabaya(PENS) \\ Jl. Raya ITS Sukolilo 60111, Indonesia \\ E-mail: rizky@pasca.student.pens.ac.id, \{puja,amang\}@pens.ac.id
}

\begin{abstract}
Multiple-Input Multiple-Output Orthogonal Frequency Division Multiplexing (MIMO-OFDM) is the most used wireless transmission scheme in the world. However, its security is the interesting problem to discuss if we want to use this scheme to transmit a sensitive data, such as in the military and commercial communication systems. In this paper, we propose a new method to increase the security of MIMO-OFDM system using the change of location of fake subcarrier. The fake subcarriers' location is generated per packet of data using Pseudo Random sequence generator. The simulation results show that the proposed scheme does not decrease the performance of conventional MIMO-OFDM. The attacker or eavesdropper gets worse Bit Error Rate (BER) than the legal receiver compared to the conventional MIMO-OFDM system.
\end{abstract}

Keywords: MIMO-OFDM, Covert Communication, Fake Subcarrier

\section{INTRODUCTION}

Security has always been a part of most discussed data transaction and communication system, however it gained more importance along with the increasing use of wireless communication systems, especially for systems in military and commercial applications. This military and commercial sometime use wireless system as its communication system. However, the main problem is that the wireless signal can be easily received by someone who has the antenna that work in the same frequency with the transmitter which transmits the signal [1]. This leakage can be fatal for communication system which sends very sensitive data.

In the wireless data transmission, there are a lot of bad effects which influence the shape of transmitted signal. These effects will cause the receiver fails to demodulate the signal. OFDM offers solution to withstand these effects. OFDM is multicarrier modulation technique [2]. It is based on the principle that by dividing the streams of data into many lower rate information streams, the speed of data transmission can be increased and also can change the shape of single frequency selective channel into a group 
of parallel flat channel. Also, OFDM can withstand the Inter-Carrier Interference (ICI) phenomenon which is happened in numerous another multicarrier modulation schemes, because of the use of frequency offset. Guard Interval (GI) and Cyclic prefix (CP) are used to combat another wireless channel problem called Inter-Symbol Interferences (ISI). Because of those advantages, OFDM, currently, becomes the widely used wireless transmission modulation and multiplexing method. The applications of OFDM are many and varied which cover Digital Broadcasting, Internet Access, Wireless Network, and Mobile Communications.

Another issue in wireless communication is the presence of obstructions in wireless channel, between transmitter and receiver. These obstructions make reflections of transmitted signal, called multipath effect, which can cause the failure of receiver to receive and demodulate the signal. The solution that has been researched to overcome this effect is by using Multiple-Input Multiple-Output (MIMO) system [3]. By combining this technique with OFDM system, the new system called MIMO-OFDM is. This technique uses the spatial multiple channel for data transmission and receptions, which can increase the BER performance and the speed of data transmission.

Recently, security of OFDM system has been widely researched [4-8]. Most of them are done by implementing cryptographic algorithm in application layer. The development of computer technology, recently, makes the increase of computing capability and may lead to increase of probability that the encrypted data can be decrypted by unauthorized person. Another solution of this leak is by modifying the physical layer of the system. In the physical layer, the transmitted signal is not the real data, but modulated symbols that represent the data and also noise, so that noise can be an additional difficulty for one who wants to attack the system.

Covering the communication is one of the methods to increase the security in the communication system. The main purpose of covert communication is to hide the informations so that the unauthorized person cannot get them. In OFDM system, this can be done in many ways. One of them is in the application layer, which is called steganography [4]. Another method that has been researched recently is hiding information in physical layer [11]. This research also proposes another alternative of covering communication system in physical layer.

\section{RELATED WORKS}

Research conducted by Hijaz et al [9] found that there are many subcarriers in OFDM standards which are not used. Hijaz implemented these subcarriers to cover another subcarrier and make camouflage subcarrier. These subcarriers configuration is constant and do not change over time. Research conducted by $\mathrm{Ma}$ et al [10] applied the constellation rotation of OFDM System to covert the channel. Ma applied a pseudorandom generator to make a random like rotation of constellation. This rotation is changed over 
time. This system was already proved in simulation that non authorized receiver cannot receive the data. Classen et al [11] conducted a research to compare the covert channel systems proposed by Hijaz and Ma. Classen did the research in practical implementation using Wireless Open-Access Research Platform (WARP). Classen tried to mimic IEEE 802.11 a standard. The practical results showed that the two systems do not influence in BER performance.

In this research we proposed the camouflage subcarrier which is changed over time using pseudorandom generator. We improve the security method proposed by Classen, Hijaz, and Ma by changing the shape of signal over time with the help of pseudorandom generator. We also try to mimic the most common used MIMO-OFDM standard, IEEE 802.11n.

\section{ORIGINALITY}

In this paper, we propose the new covert communication scheme for MIMO-OFDM system at the physical layer by inserting the fake subcarriers and changing the location of them by using the pseudorandom generator. The MIMO-OFDM system used in this paper mimic the specifications of the WLAN 802.11n. Pseudorandom generator is deployed in transmitter and the receiver side. The detail system of Pseudorandom generator is known by the transmitter and receiver. To start the Pseudorandom Generator as well as the MIMO-OFDM system, the transmitter and receiver have to know the initial start key. This key is already defined inside the transmitter and receiver system and need to be same.

\section{SYSTEM DESIGN}

\subsection{MIMO-OFDM System}

We tried to mimic the MIMO-OFDM 802.11n standard for this research. This system consists of two antennas in transmitter side and two antennas in the receiver side. The number of Fast Fourier Transform (FFT) used is 64. This number will make the MIMO-OFDM have $20 \mathrm{MHz}$ bandwidth. The channel estimation used in this scenario is Minimum Mean Square Error (MMSE). Table 1 shows the specific MIMO-OFDM parameters used in this research.

The transmitter process starts from the generation of bits representing the data. Then data are encoded using convolutional encoder with the number of rate is $1 / 2$. In this step, the encoded data is known as codewords. The codewords are modulated using Quadrature Amplitude Modulation (QAM). The result of this process is called data symbol. After that, the data symbol will be configured to make subcarriers of OFDM. The number of subcarrieris equal to FFT size. In 802.11n standard, the number of FFT is 64 , which 52 of them can be used as data. The subcarrier number -26 until -1 and +1 until +26 are used for data symbol. The subcarrier number 0 is for center carrier. 
Table 1. MIMO-OFDM Simulation Parameters

\begin{tabular}{|c|c|}
\hline Parameters & Value \\
\hline Carriers Frequency & $2.4 \mathrm{GHz}$ \\
\hline Bandwidth & $20 \mathrm{MHz}$ \\
\hline Channel Encoder & Convolutional Code \\
\hline Modulation & 4-QAM \\
\hline $\begin{array}{l}\text { Number of Data } \\
\text { Subcarrier }\end{array}$ & 52 \\
\hline FFT Size & 64 \\
\hline Cyclic Prefix Length & $1 / 4$ \\
\hline $\begin{array}{l}\text { Signal to Noise Ratio } \\
\text { Range }\end{array}$ & $0-25 \mathrm{~dB}$ \\
\hline Channel Model & 2-path Rayleigh Fading \\
\hline Number of Antennas & $\begin{array}{c}2 \text { in Transmitter, and } 2 \\
\text { in Receiver }\end{array}$ \\
\hline Channel Estimation & MMSE \\
\hline MIMO Detectors & MMSE \\
\hline
\end{tabular}

The subcarrier numbers which are not used in conventional MIMOOFDM basicly will be inserted with zero padding. This unused subcarrier will be reserved for encryption process which is described later. Pilot symbol is inserted after zero padding insertion process. This pilot symbol is applied to estimate the channel effect. The pilot symbol configuration in this research is High Throughput Long Training Field (HT-LTF). After the symbols are packeted, the packet then will be parallelized and processed with IFFT. The result of this process is referred to as OFDM symbol. The next process is inserting the Cyclic Prefix (CP). After the CP inserting process, OFDM symbol then is serialized and transmitted using antenna.The transmitted signal can be expressed as (1).

$$
y=\operatorname{IFFT}_{N}(x)
$$

Where $y=\left[y_{0}, y_{1}, y_{2}, \ldots, y_{N-1}\right]$ is the transmitted signal and $x=\left[x_{0}, x_{1}, x_{2}, \ldots, x_{N-1}\right]$ is packetized data symbol. The full process of MIMOOFDM system in transmitter side is showed in Figure 1.

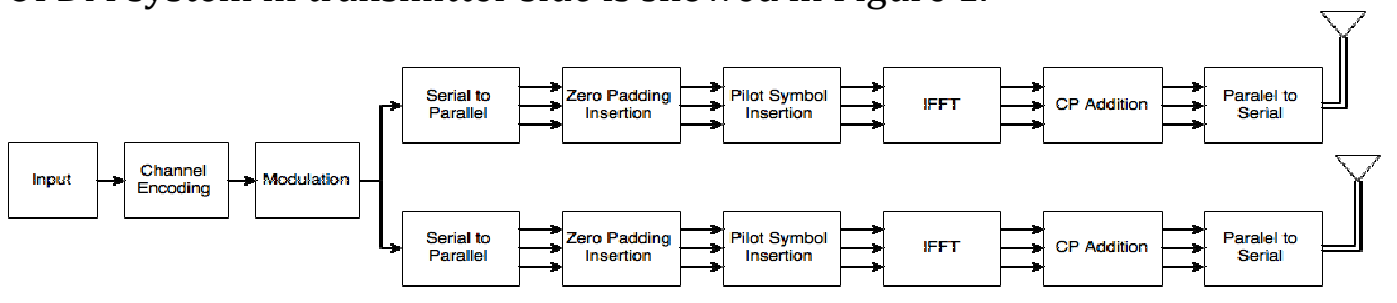

Figure 1. MIMO-OFDM System with 2 Antennas in Transmitter Side 
We consider that MIMO-OFDM is applied in the uplink, where the signals from users occupy different channels. In this research, channel model used is Rayleigh Fading Channel Model (RFCM). RFCM is a kind of rational model when a lot of objects in the environment caused transmitted signal scatter before it comes to receiver side. The RFCM model is expressed in mathematical symbol as (2).

$$
h=a+j b
$$

Where $h=\left[h_{0}, h_{1}, h_{2}, \ldots, h_{N-1}\right]$ is the channel response. $a=\left[a_{0}, a_{1}, a_{2}, \ldots, a_{N-1}\right]$ is gaussian random real part and $b=\left[b_{0}, b_{1}, b_{2}, \ldots, b_{N-1}\right]$ is the imaginary part. This channel model consist of real and imaginary part.

In the receiver side, the process starts with receiving transmitted signal from the antenna. The received signal is expressed as (3).

$$
y_{k}=x_{k} h_{k}+n_{k}
$$

Where $y$ is received signal, $x$ is transmitted signal, $\mathrm{h}$ is channel response, and $\mathrm{n}$ is noise vector, with $k=[0,1,2, \ldots, N-1]$. The next process is Cyclic Prefix (CP) removal. The $\mathrm{CP}$ is extracted from the received signal. After that, the signal is ready for FFT process. After FFT process, the pilot symbol is extracted. The extracted pilot symbol then is processed to estimate the channel using MMSE channel estimation method. The channel estimation process can be expressed as (4).

$$
g_{M M S E}=R_{g y} R_{Y Y}^{-1} y
$$

Where $R_{g y}$ is cross-correlation matrix between matrix $\mathrm{g}$ and matrix $\mathrm{y}$, $R_{g y}=E\left(g y^{h}\right)$, while $R_{y y}$ is auto-correlation matrix from matrix y, $R_{y y}=E\left(y y^{h}\right.$ ). To calculate the estimation value of channel matrix, (5) is used.

$$
\begin{aligned}
& h_{M M S E}=F g_{M M S E}=F Q_{M M S E} F^{H} X^{H} y \\
& \text { with } \\
& Q_{M M S E}=R_{g g}\left[\left(F^{H} X^{H} X F\right) \delta_{n}^{2}+R_{g g}\right]^{-1}\left(F^{H} X^{H} X F\right)^{-1}
\end{aligned}
$$

F is orthogonal FFT matrix, and the noise variance $E\left\{(|n|)^{2}\right\}$ is expressed as $\delta_{n}^{2}$.

After $h_{\text {MMSE }}$ is calculated, the next process is to detect the data using MMSE detection methods. This detector is used to detect the signal and combine the separated signal from two transmitter's antenna. MMSE eliminate the interference and noise by minimizes the mean square error from the estimated channel matrix, but, the receiver must have the information about Signal to Noise Ratio (SNR). The detector method which is used in this research can be expressed as (7). 


$$
\hat{x}=\left(H^{H} H+\delta_{n}^{2}\right)^{-1} H^{A} \bar{y}
$$

Where $\hat{x}$ is detected received signal, $H$ is estimated channel matrix, in this research, $H$ is equal to $\bar{h}_{\text {MMSE}}$, and $\bar{y}$ is undetected received signal. $H^{H}$ represents the pseudo-inverse of $H$ and $\delta_{n}^{2}$ is Signal to Noise Ratio (SNR).

The last process of MIMO-OFDM's receiver side is demodulation and decoder. Demodulation is done by QAM demapper. This demodulation process turns the symbol back to bits of information. After that, convolutional decoder is applied. Finally, the bits then will turn back to information message. The full process of MIMO-OFDM receiver is presented in Figure 2.

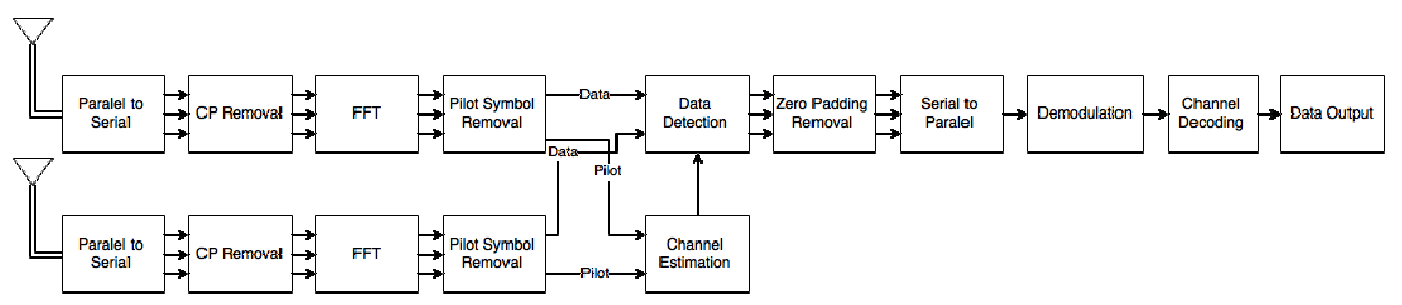

Figure 2. MIMO-OFDM System with 2 Antennas in Receiver Side

\subsection{Pseudo Random Location of Fake Subcarrier}

We already analyzed the subcarrier configuration in OFDM standard and found that not all subcarriers in OFDM standard are used. Figure 3 shows the subcarrier configuration of IEEE 802.11n standard.

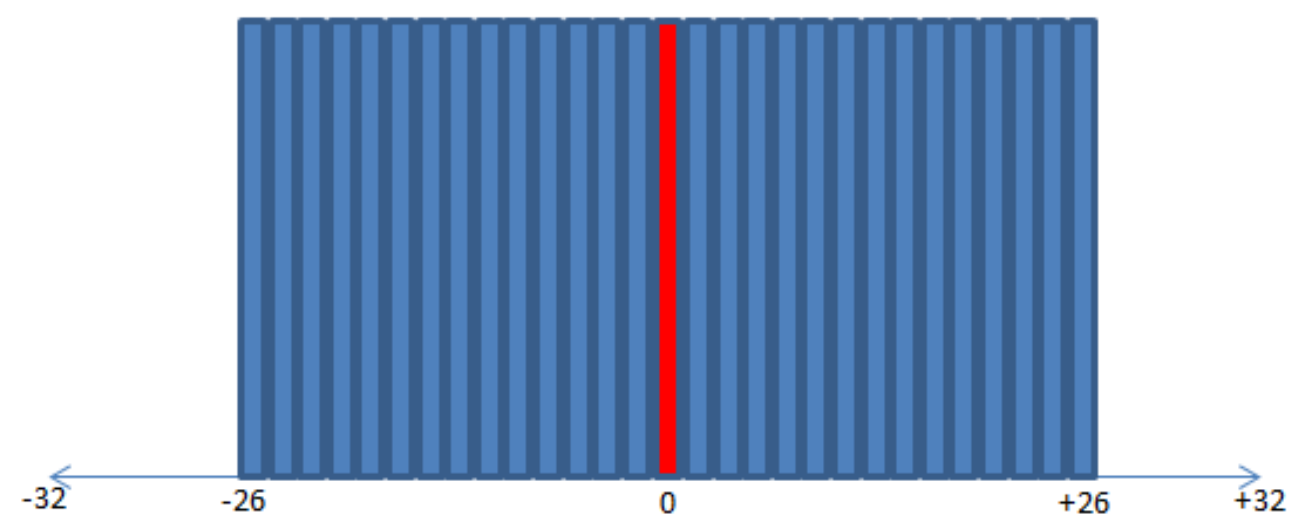

Figure 3. Subcarrier configuration of IEEE 802.11n

As presented in Figure 3, subcarrier 0 is the center of frequency, and the subcarrier -1 to -26 and +1 to +26 are used for data. Subcarrier -27 to -32 and subcarrier +27 to +32 are not used. Basicly, subcarrier -27 to -32 and +27 to +32 is inserted by zero. This zero insertion in OFDM subcarrier is known as zero padding. Zero padding can reduce complexity of OFDM FFT process, but in this research, we assumed that the security is more important than the data transmission speed. 
The basic idea of the proposed method in this research is that the receiver cannot receive if the subcarrier configuration is wrong. From this idea, we make a new configuration of subcarrier for OFDM system, especially for IEEE $802.11 \mathrm{n}$, where this configuration will change automatically over time based on the code generated from pseudorandom generator.

\subsubsection{Galois Linear Feedback Shift Register (GLSFR)}

This proposed system starts by generating the code which represents the location of a fake subcarrier. For generating the random code, we use Galois Linear Feedback Shift Register (GLFSR). In choosing the good random sequence generator,we should consider how many periods that the generator can provide. The bigger period is better but we should also consider how many bits that will be used to represent the location of fake subcarrier. The maximal number of bits that can be used is equal to the number of subcarrier. Figure 4 shows the 4-bit GLFSR which is used for the simulation process.

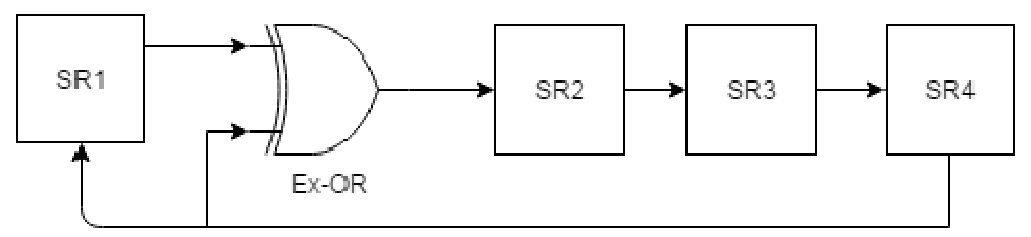

Figure 4. 4-Bit GLFSR Pseudo Random Generator

The generated bits from GLFSR pseudorandom generator then is converted to decimal form. This decimal form will represent the location of fake subcarrier. SR1 generate the positive or negative value of the subcarrier location while SR2, SR3, and SR4 generate the location number of fake subcarrier. To make a hole for this fake subcarrier, the subcarrier which already filled with data need to be shifted. The shifting process needs to follow two of these rules. First, the DC component in the center of subcarrier (subcarrier 0) cannot be shifted, and must be still in that position. Second, the right side (positive side of subcarrier) and the left side (negative side of subcarrier) must have the same number of subcarrier. These rules are made by us to maintain the shape of subcarrier configuration and to keep the advantages of OFDM without reducing its performance. According to the rules, we made this encryption steps.

Step 1:

Initial state of register is inserted. The initial state of register must be the same for transmitter and receiver side to generate the same random sequence.

Step2:

The output of registeris converted to decimal form which represents the location of first fake subcarrier. To make the number of subcarrier in left and right side equal, the second subcarrier is added before (for positive location) or after (for negative location) first subcarrier. The subcarrier before and after the fake subcarriersare then shifted one step. The subcarriers before 
fake subcarriers are shifted left and subcarriers after fake subcarriers are shifted right. Subcarrier 0 is not shifted and kept in its position. Figure 4 shows the subcarrier configuration if the random generator output is 15 .

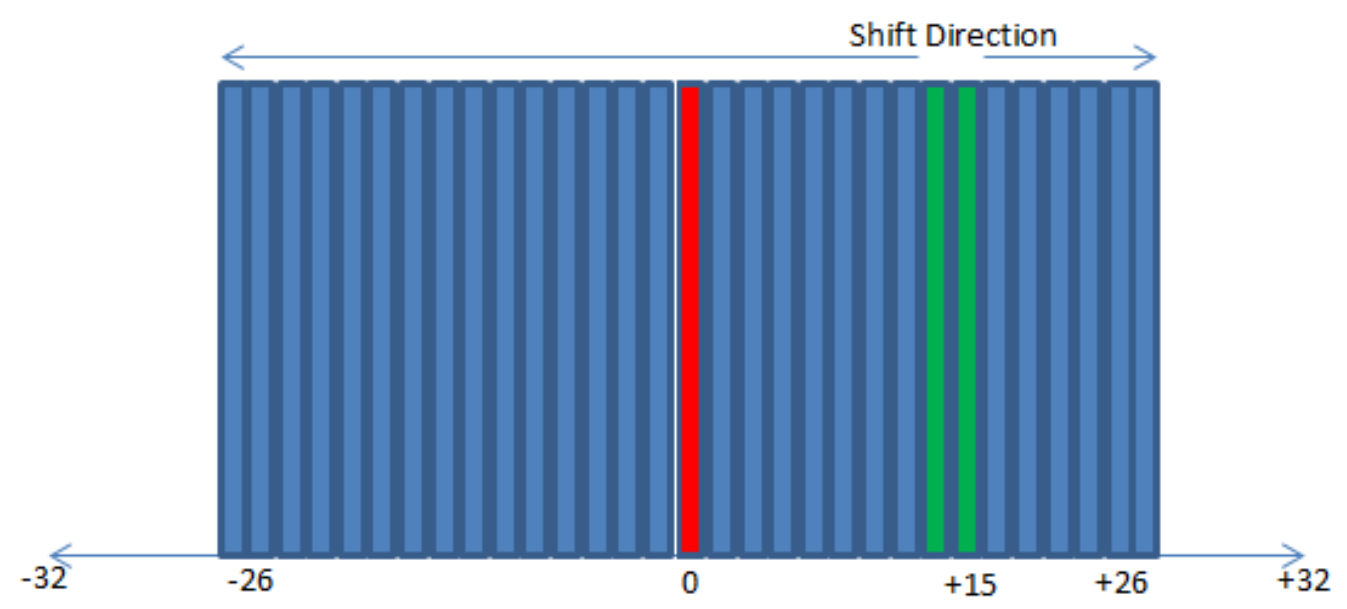

Figure 4. Subcarrier configuration of IEEE $802.11 \mathrm{n}$ with camouflage subcarrier

The green subcarriers in Figure 4 are the fake subcarriers. This subcarrier configuration with fake subcarrier inserted is referred to as camouflage subcarrier. All of the positions generated by GLSFR is showed by figure. The initial value of this scheme is 1000 .

Table 2. Fake Subcarrier Position Generated by GLSFR

\begin{tabular}{|c|c|c|c|}
\hline Time & First Value & Next Value & $\begin{array}{c}\text { Fake Subcarrier } \\
\text { Position }\end{array}$ \\
\hline 1 & 1000 & 0100 & 4 \\
\hline 2 & 0100 & 0010 & 2 \\
\hline 3 & 0010 & 0001 & 1 \\
\hline 4 & 0001 & 1100 & 12 \\
\hline 5 & 1100 & 0110 & 6 \\
\hline 6 & 0110 & 0011 & 13 \\
\hline 7 & 0011 & 1101 & 10 \\
\hline 8 & 1101 & 1010 & 14 \\
\hline 9 & 1010 & 0101 & 15 \\
\hline 10 & 0101 & 1110 & 11 \\
\hline 11 & 1110 & 1111 & 9 \\
\hline 12 & 1111 & 1011 & 4 \\
\hline 13 & 1011 & 1001 & \\
\hline 14 & 1001 & 1000 & 0100 \\
\hline 15 & 1000 & & \\
\hline
\end{tabular}

In the receiver side, the process to remove the fake subcarrier is the counterpart of the mentioned steps. Using the same initial state and same 
generator with transmitter side, the receiver can detect the location of first and second fake subcarrier, and then shift back another subcarrier to its real location. Figure 5 shows the MIMO-OFDM transmitter and receiver block diagram with pseudorandom generator, fake subcarrier inserter, and remover.

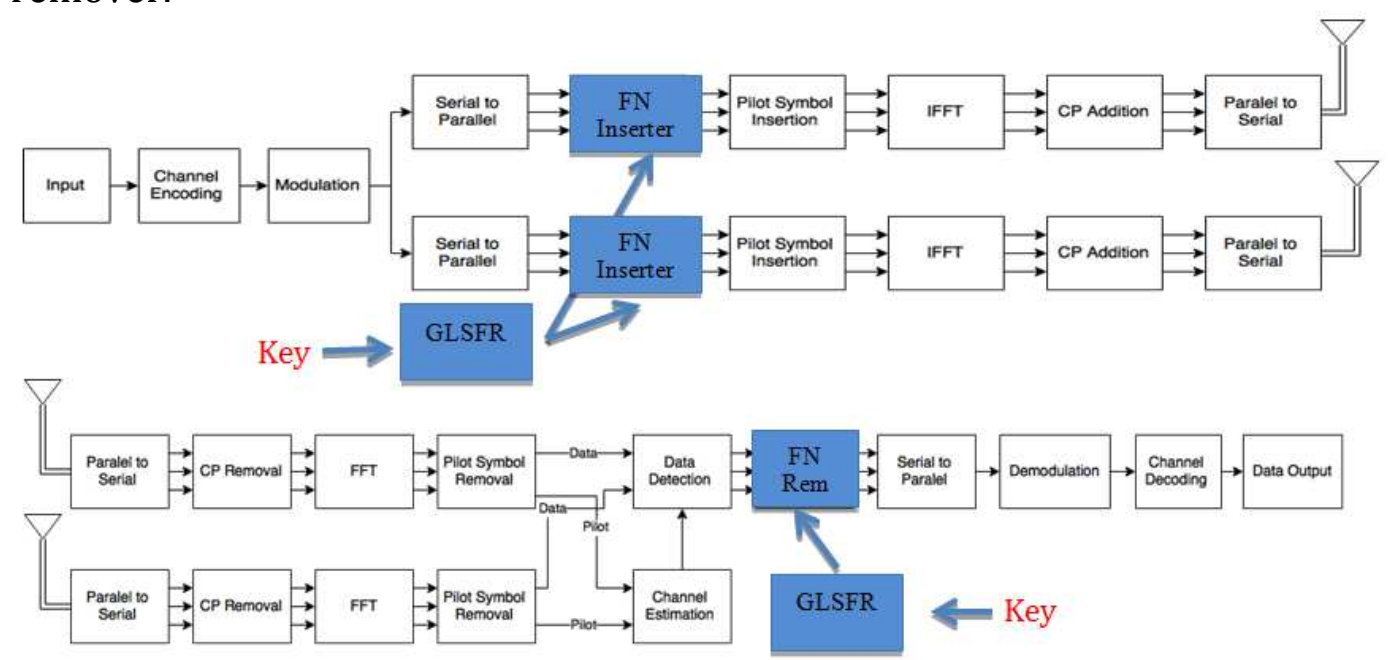

Figure 5. Transmitter and Receiver Side with Fake Subcarriers (FS) System

\section{EXPERIMENT AND ANALYSIS}

The first question addressing to this system is the BER performance of the system using covert channel and non covert channel. To answer this question, we provide simulation result of BER performance between MIMOOFDM with proposed covert and conventional non-covert system [13]. The simulation process is done by using the MIMO-OFDM parameters stated in Table 1. Figure 6 shows that the BER performance between covert system and non-covert system are equal.

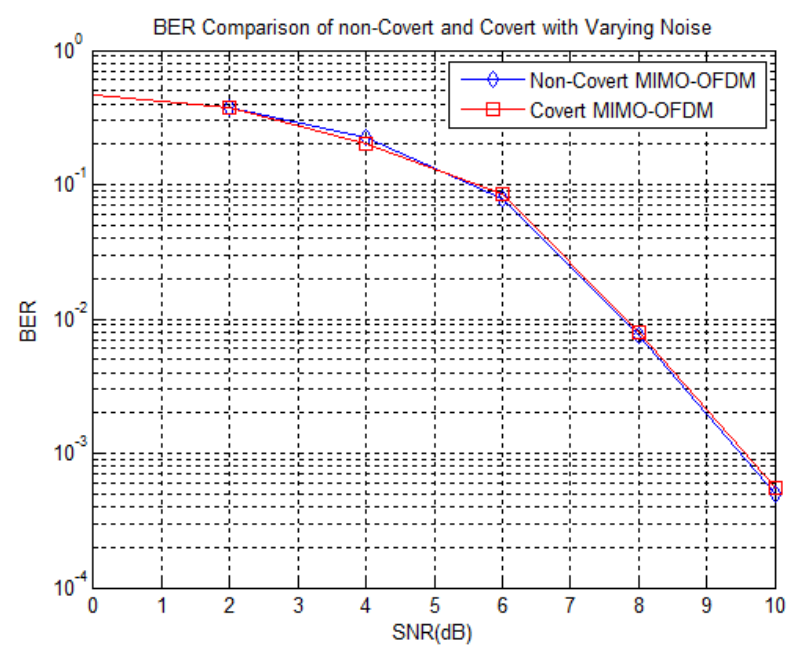

Figure 6. IEEE 802.11n Transmitter and Receiver Side with Camouflage Subcarrier 
The next simulation is for investigating the shape of MIMO-OFDM signal between covert and non covertsystem. Figure 7 shows that there is only a small difference between them. If this signal is transmitted very fast, the differences between them will be hard to see.

The last simulation is provided to investigate the signal received by authorized receiver and non authorized receiver. We assume that the nonauthorized receiver does not know the random shifting scheme in MIMOOFDM subcarriers and only authorized receiver knows this scheme. We also assume that the transmitter and receiver already know the same initial state to start the GLFS Register to generate the random location of fake subcarrier. Figure 8 shows scenario of the non-authorized receiver try to eavesdrop the communication between the authorized transmitter and authorized receiver. We simulated this scenario, and the result is showed in Figure 9.

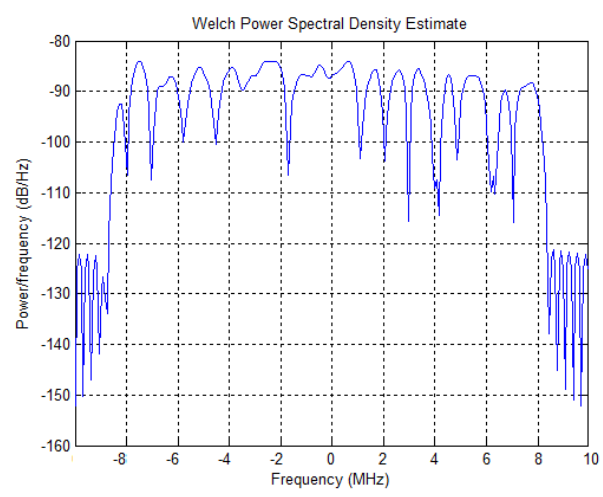

(a)

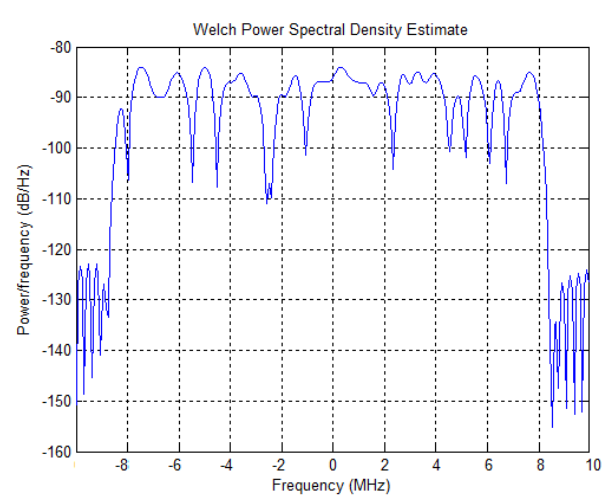

(c)

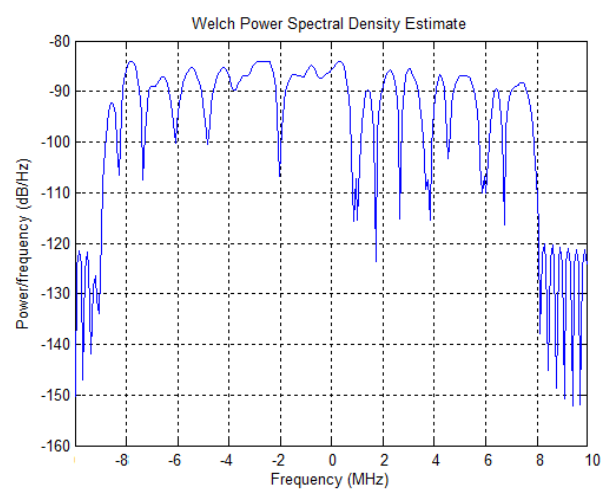

(b)

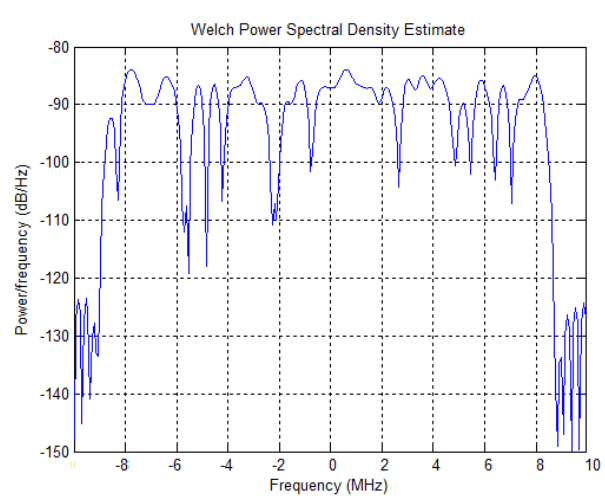

(d)

Figure 7. The PSD of OFDM Signal for (a) Non-covert system, (b) covert system with fake subcarrier in subcarrier +15 , (c) non-covert system with different transmitted bit, and (d) covert system with fake subcarrier in subcarrier -2 


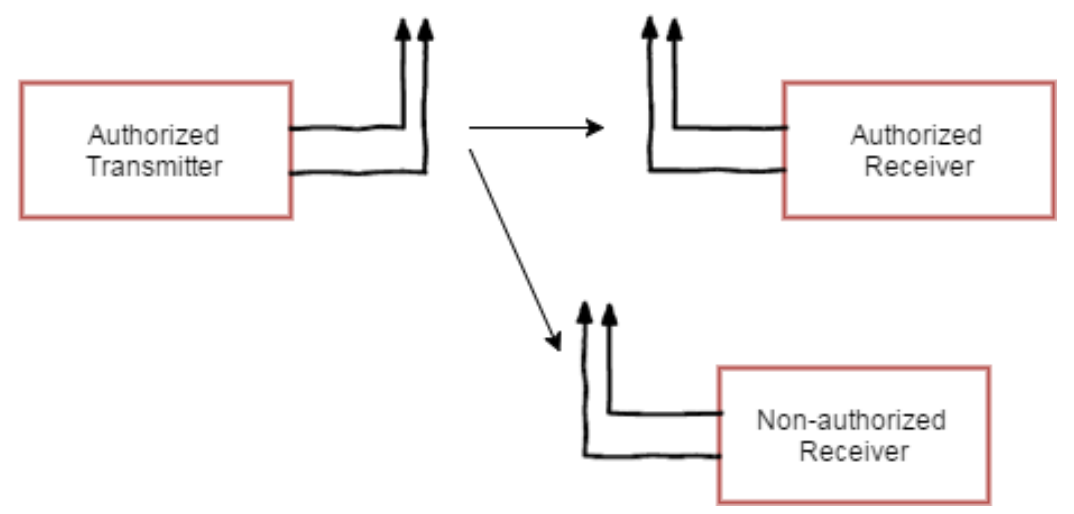

Figure 8. Scenario Used for Simulation Process

The BER in Figure 9 shows that the unauthorized receiver only gets the BER of around 0.6. It means almost a half of the received signal is not correct. The authorized receiver gets better BER than the unauthorized receiver which means the authorized receiver gets the information. From this result, we prove that the secure communication system is guaranteed.

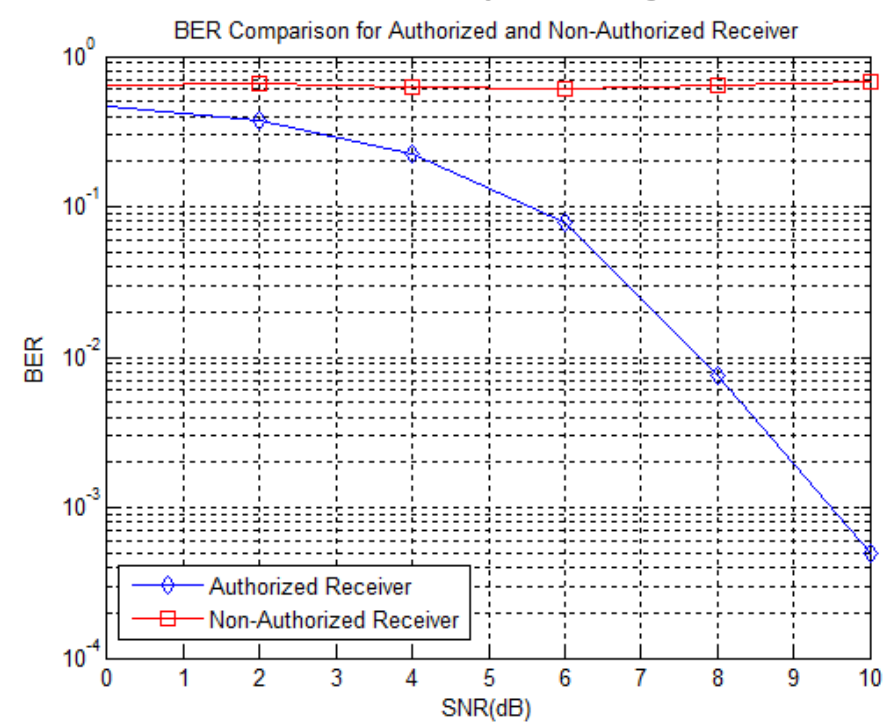

Figure 9. BER Performance between Authorized Receiver and Non Authorized Receiver

\subsection{The Comparative Analysis between Proposed and The Other Schemes}

The fair comparison between proposed scheme and other schemes in similar area is demanding, since they behave differently depending on where they implement the security method. Some covert channels are frame based [10] while the others are symbol based [9][11]. According to the comparison analyzed by Classen, frame based scheme has better performance in wireless channel with strong changing amplitude, in the other hand, symbol based 
scheme has better performance in channel with a lot of frequency or time mismatch. This is happened because the frame based scheme modifies the frame by inserting something to the packet of data. This insertion and calculation do not affect the modulation schemes, so it will not be affected by rapidly changing amplitude in wireless channel. The disadvantage of frame based scheme is that it is very sensitive to time mismatch, because it does a calculations in received packet data and sometimes can cause a delay. The symbol based scheme has better performance in channel with frequency or time mismatch because this scheme only modifies the modulation process and does not modify the packet of data. The covert scheme proposed by Hijaz is frame based, while the scheme proposed by Ma is symbol based.

We also investigate the detection probability between proposed sceme and other schemes. Table summarizes the detectability of all covert schemes in OFDM system. The camouflage subcarrier proposed by Hijaz can be detected if the unauthorized receiver take a look at the spectrum by using spectrum analyzer. The constellation rotation proposed by Ma cannot be detected at the spectrum but can be easily detected by looking at IQ constellation diagram. Classen's CP replacement method uses modified CP to cover the transmission. This will reduce the performance of OFDM system because $\mathrm{CP}$ is essential in detecting the packet start and stop. Classen's Carrier Frequency Offset (CFO) modification also has the similar problem with CP replacement. Both of them modify the essential synchronization system of OFDM. However, all of the covert channel scheme can be detected by looking at IQ constellation. Furthermore, the data can be extracted if the signal is succesfully decoded and checked. Our proposed scheme has a pseudorandom location of fake subcarrier. It means it will be hard to decode because the location of fake subcarrier is always moving.

Table 3. Detectability Comparison: detectable (y), non detectable (n), performance trade of (p)

\begin{tabular}{|l|c|c|c|c|c|}
\hline \multicolumn{1}{|c|}{ Layer } & $\begin{array}{c}\text { Hijaz's } \\
\text { Camouflage } \\
\text { Subcarrier }\end{array}$ & $\begin{array}{c}\text { Ma's Rotating } \\
\text { Constellation }\end{array}$ & $\begin{array}{c}\text { Classen's CP } \\
\text { Replacement }\end{array}$ & $\begin{array}{c}\text { Classen's CFO } \\
\text { Modification }\end{array}$ & $\begin{array}{c}\text { Our } \\
\text { Proposed } \\
\text { Method }\end{array}$ \\
\hline Spectrum & $\mathrm{y}$ & $\mathrm{n}$ & $\mathrm{y}, \mathrm{p}$ & $\mathrm{n}$ & $\mathrm{n}$ \\
\hline Constellation & $\mathrm{n}$ & $\mathrm{y}$ & $\mathrm{n}$ & $\mathrm{y}$ & $\mathrm{n}$ \\
\hline BER & $\mathrm{n}$ & $\mathrm{n}$ & $\mathrm{y}, \mathrm{p}$ & $\mathrm{p}$ & $\mathrm{n}$ \\
\hline
\end{tabular}

\section{CONCLUSION}

In this paper, the fake subcarrier insertion method in MIMO-OFDM system is proposed, whereby the pseudorandom generator is used to change the location of fake subcarrier. This pseudorandom generator enhances its security level. The unauthorized receiver cannot receive the signal due to the unknown information of initial state key of pseudorandom generator. There are only small differences between covert and non covert MIMO-OFDM 
signal, which means that the OFDM performance will not be decreased if the covert system is applied. In the future work, we will try to implement this method in real device using Universal Software Radio Peripheral (USRP). We will analyze this method in real condition with real wireless channel environment.

\section{Acknowledgements}

This research is granted by PoliteknikElektronikaNegeri Surabaya (PENS) Freshgraduate Scholarship for Master's Degree Students.

\section{REFERENCES}

[1] Y. Liang H., V. Poor, S.Shamai, Secure Communication over Fading Channel,IEEE Transactions of Information Theory, Vol.54, No. 6, pp. 2470-2492, 2008.

[2] Z. Wang, X. Ma,G. B. Giannakis, OFDM or Single Carrier Block Transmission?,IEEE Transactions on Communications, Vol.52, No. 3, pp. 380-394, 2004.

[3] G.L. Suber, J. R. Barry, S. W. Mclaughlin, and Ye Li, Broadband MIMOOFDM Wireless Communications, Proceedings of the IEEE, Vol. 92, No. 2, pp. 271-294, 2004.

[4] S. Grabski, K. Szczypiorski, Steganography in OFDM Symbols of Fast IEEE 802.11n Networks, IEEE In Security and Privacy Workshops (SPW),San Francisco,pp. 158-164, 2013.

[5] C. Kratzer, J. Dittman, A. Lang, T. Kuhne, WLAN Steganography: First Practical Review,Proceedings of The $8^{\text {th }}$ Workshop on Multimedia and Security,New York, pp. 17-22, 2006.

[6] C. Kratzer, J. Dittman, R. Merkel, WLAN Steganography Revisited,In Proceedings of SPIE Electronic Imaging,San Jose, pp. 68-70, 2008.

[7] X. Tan, K. Borle, W. Du, B. Chen, Cryptography Link Signature for Spectrum Usage Authentication in Cognitive Radio, In Proceedings of The Fourth ACM Conference of Wireless Network Security, San Jose, pp. 79-90, 2011.

[8] M. Willhelm, I. Martinovic, J. B. Schmitt, V. Lenders, WiFire: a Firewall for the Wireless Network, In Proceeding of the ACM 2011 SIGCOMM, Toronto, pp. 456-457, 2011.

[9] Z. Hijaz, V. Frost, Exploiting OFDM Systems for Covert Communication, In Military Communications Conference, pp. 21492155, 2010.

[10] R. Ma, L. Dai, Z. Wang, Secure Communication in TDS-OFDM Using Constellation Rotation and Noise Insertion, IEEE Transactions on Consumer Electronics, Vol. 56, No. 3, pp. 1328-1332, 2010.

[11] J. Classen, M. Schulz, M. Hollick, Practical Covert Channels for WiFi Systems, IEEE Conference on Communications and Network Security (CNS), Florence, pp. 209-217, 2015. 
[12] The Institute of Electrical and Electronic Engineers, Inc.,IEEE Standard of Information Technology,IEEE Press (New York), 802.11-2012, 2012.

[13] R. Raissawinda, I. G. P. Astawa, Y. Moegiharto, A. Zainudin, I. D. Agusalim, Channel Estimation Design of MIMO-OFDM Systems using MMSE for IEEE 802.11n Standard, European Scientific Journal, Vol. 10, No. 6, pp. 138-146, 2014. 